\title{
Leprosy and Cancer
}

\section{Hague $A^{*}$}

CellSonic: Manufacturers of Medical Equipment, United Kingdom

*Corresponding author: Andrew Hague, President, CellSonic: Manufacturers of Medical Equipment, United Kingdom, Tel: +1 315210 6307; Email:

\section{Editorial}

Volume 3 Issue 1

Received Date: January 28, 2019

Published Date: February 05, 2019

DOI: $10.23880 /$ eoij-16000195

cellsonic.beauty@gmail.com

\section{Editorial}

These two diseases share notoriety and can be fatal. Thankfully they are now easy to cure; leprosy since 1946 with dapsone and cancer since 2017 with CellSonic VIPP.

Leprosy is a chronic, mildly infectious disease caused by Mycobacterium leprae, an acid-fast, rod-shaped bacillus [1]. Around 95 percent of the world's population is naturally immune to leprosy even after prolonged exposure. Despite this, communities may still ostracise a sufferer which leads many with the disease to avoid diagnosis for fear of isolation.

The extent of this discrimination is seen in the advice that it is offensive to call a person a leper [2]. The word leper has come to mean outcast. The terms "person affected by leprosy" and "Hansen's disease" are used instead.

In 1981, a World Health Organisation Study Group developed a multi-drug therapy (MDT) of dapsone and rifampicin for all patients with Clofazimine added for multi-bacillary disease to kill the pathogen and cure the patient. This is provided free of charge by Novartis who have agreed to give it until 2020 [3].

A report from 1981 of 2,383 Japanese patients with leprosy in a leprosarium in Japan shows them also suffering from tuberculosis, pneumonia and bronchitis, nephritis and nephrosis [4]. The suicide rate was high among female patients. Deaths from total malignant neoplasms were higher than expected among patients with lepromatous leprosy for both sexes. Mortalities from cancers of the cervix and the oesophagus among females with lepromatous leprosy were significantly high. It is hoped that since the 1980s diagnosis is better bringing treatment sooner to avoid the deterioration that allows other diseases to enter.

The disfigurement caused by leprosy of the skin and extremities of limbs can be treated by CellSonic combined with the multi-drug therapies.

The figure shows gangrene on a 90-year-old lady being treated with CellSonic VIPP (very intense pressure pulses) [5]. Vascularisation is improved so that under the dead outer layer of skin, new tissues form leaving the external layer as a protection to fall away when the newly formed skin can be safely exposed.

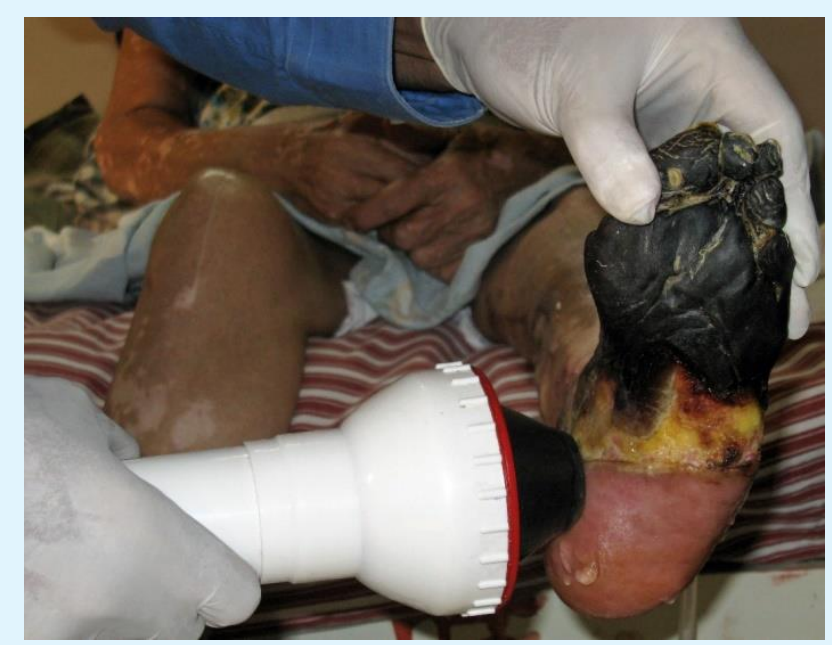

Figure 1: 90-year-old lady being treated with CellSonic VIPP. 


\section{Ergonomics International Journal}

Cancer is not infectious and generates considerable sympathy for the sufferers. Contracting leprosy is unfortunate despite the advice to avoid close contact with the afflicted. Getting cancer, some argue, is a result of not living a healthy life. That is an unfair criticism based on the assumption that cancer is avoidable by having an organic diet, lots of exercise and no stress. The reality is that there is a statistical inevitability of cancer. All the time, the cells of our bodies are replacing themselves and new copies should be the same as the old ones but with billions of cells dividing the chances are that a few cells will not be exact copies. They will be different and that change is called a mutation. The changed cells can go on to replicate more changed cells and these we call cancer. Nature depends on mutations. Without them, humans could never have evolved.

To repair the damage of cancers, we have an immune system. It detects cancers and kills them before they have multiplied. At least that's what should happen for healthy people eating good food, exercising and sleeping well. Unfortunately, life seldom runs perfectly and the immune system's performance may be poor allowing the cancer to escape and grow.

To understand what causes cancer, look at what impairs the immune system. Smoking tobacco is damaging beyond doubt. The role of tobacco companies in causing death is a significant part of the history of the $20^{\text {th }}$ century and must stand alongside other mass killers and warfare. Alcohol is similarly dangerous and as this too is a profitable industry escapes condemnation and is treated as though it only causes temporary behaviour failures rather than permanent organ damage. Remember that medical studies will only be done when the research is sponsored and big payers are never going to advice against big money. Similarly, the risks of brain damage from smartphones are ignored. Research at Bradford University in England has concluded that the maximum time for a smartphone to be held safely against the ear is six minutes; beyond that the brain is cooking. Electricity transmission generates powerful electro-magnetic fields that disrupt the stability of the electrical properties in all living creatures, not just humans. Only when large power stations are replaced by smaller, local generators will this disruptive factor, hitherto largely ignored as a health problem, be eliminated.

Fashion dictates that sunglass should be worn outdoors. This prevents the eyes learning the true brightness of the sun so that the skin is not naturally protected. Perhaps this is mitigated by the other fashion trend to block all sunlight from the skin with barrier creams and make up. The end result deprives the immune system of essential vitamin D for which synthetic food additives are no substitute. Without vitamin $\mathrm{D}$, the immune system is weak and no amount of avoiding unhealthy food will compensate. Few people in modern, urban communities have an outdoor life. This is more of a problem for dark skinned people because their pigmentation slows down the rate at which vitamin $\mathrm{D}$ is taken in $[6,7]$.

As society has become fragmented with more geographical and social mobility, stress has increased and this seriously restricts the immune system. The ideal of living in the group into which one was born is not available in modern society. The importance of exercise for a majority of people has been lost. They travel and work sitting down and seldom burn as many calories as they consume. If the goal is an easy life, it is not achieved without vigorous muscle action.

Cancer is not a bio-chemical disease. The electrical properties of cancer cells are well understood and described by Dr Steve Haltiwanger in his classic paper [8]. Studies at Bradford University in England have shown that cancer tissues have different permittivity values to healthy cells, 9.6 for cancer and 3.2 for healthy tissue with nothing in between.

This observation explains the success of CellSonic VIPP switching cancer cells from mutating to exact replicating, in other words, stopping cancer [9]. The nonsurgical, irreversible electroporation has an immediate effect when the pulses from the CellSonic head hit the tumour.

CellSonic is now making the cancer diagnostics into a machine to be installed in clinics so that it will be possible to diagnose and cure in twenty minutes. Over three million clinics will be needed worldwide. A cancer will be found and stopped anywhere in the body before a patient is aware of it. The plan is for everyone to be checked every six months so that no one suffers from a growing tumour. Cancers will always form. That statistical likelihood cannot be stopped. Leprosy as a parasite may one day be exterminated but cancer is inevitable for humans. Thankfully, science has found the way to stop cancer before it becomes dangerous [10].

\section{References}

1. Hague A (2014) Some ancient mysteries of leprosy uncovered. Anderson Cancer Center, University of Texas M. D, USA. 


\section{Ergonomics International Journal}

2. Leprosy Frequently Asked Questions. American Leprosy Missions.

3. Who (2018) Leprosy. World Health Organisation.

4. Shinkan Tokudome, Suminori Kono, Masato Ikeda, Masanori Kuratsune, Shigeru Kumamaru (1981) Cancer and Other Causes of Death Among Leprosy Patients, JNCI: Journal of the National Cancer Institute67(2): 285-289.

5. http://www.cellsonic-medical.com/wound.htm
6. Sun Exposure: Vitamin D and Other Health Benefits of Sunlight.

7. The Benefits of Vitamin D.

8. The Electrical Properties of Cancer Cells.

9. Hague A (2017) Review of reports of curing cancer with Cell Sonic VIPP machines. Gen Med Open.

10. Hague A (2018) Cancer's Change of Direction. J Med Res Biol Stud 1: 104. 